\title{
Regionalization of care: centralizing complex surgical procedures
}

\author{
Brent K Hollenbeck, David C Miller, John T Wei and James E Montie
}

There is growing concern among patients and those responsible for paying for health care (payers) regarding the quality of health care that is delivered in the US. Each year, a large number of potentially avoidable deaths occur in our hospitals, underscoring the need for significant improvement. The Institute of Medicine has delineated this "quality chasm" as representing the discrepancy between actual and ideal clinical practice. The seemingly pervasive nature of the quality problem has been highlighted for even the most recognizable processes of care, such as the administration of beta blockers after acute myocardial infarction, prompting both payers and health care providers to seek out mechanisms for improving quality.

Current initiatives occurring at the federal level include value-based purchasing (i.e. pay for performance) and the Centers of Excellence program, both undertaken by the Centers for Medicare and Medicaid Services. The former seeks to reward providers for providing appropriate care, and the latter attempts to direct patients, for select procedures, to institutions with a proven track record in quality. Despite this recent endorsement at the legislative level, centralizing complex surgical cases to select medical centers is not a novel phenomenon. Since the late 1970s, a number of large, population-based studies have demonstrated better outcomes at high-volume centers for a variety of surgical procedures. Centers in which complex procedures-such as coronary artery bypass grafting and major cancer resections including pancreatectomy, esophagectomy, and cystectomy-are commonly performed are more likely to possess the requisite ancillary services in order to better care for these patients. Perhaps, most importantly, the concept that high-volume hospitals and surgeons have better outcomes has face validity both with our consumers (e.g. patients and payers) and with our fellow surgeons.
With nearly 30

years of work

focusing on

the benefits

of centralizing

care...the

benefits to the

patient are too

large to ignore.

All of the authors are based at the

Department of Urology, University

of Michigan, Ann Arbor, MI, USA.

\section{Competing interests}

The authors declared

they have no competing

interests.

www.nature.com/clinicalpractice doi:10.1038/ncpuro0322
Regionalization of complex surgical care would not be without its shortcomings. The notion that concentration of care would place a significant burden on the patient, in terms of travel time, has, to some degree, been discredited. A more significant issue of regionalization would relate to its impact on small, rural medical centers. Specifically, regionalization of a large number of services to urban centers could siphon away many of the more lucrative procedures, thereby threatening the fiscal viability of rural institutions. The worst case scenario being that the fallout from patient migration could lead to rural medical center collapse, thus reducing patient access to general medical care in these regions. Conversely, regionalization to tertiary centers could substantially increase the burden on these institutions. Increasing the caseload of complex, high-risk procedures may preclude these institutions from performing lucrative low-risk procedures, potentially impacting their bottom line as well; thus, alternative reimbursement strategies that account for this increasing burden of care may be needed.

Despite these potential limitations, regionalization of care is underway for a variety of reasons. Current reimbursement climates have caused providers to shy away from performing lengthy, relatively low paying, complex surgical procedures, leading to selective referral of 'undesirable' cases to tertiary centers. Furthermore, patients and payers are becoming savvy. With nearly 30 years of work focusing on the benefits of centralizing care, the time has come to operationalize this body of work; the benefits to the patient are too large to ignore. Although the current model for regionalization is unstructured, new initiatives, such as the Centers of Excellence and value-based purchasing approaches by Medicare, and increasing payer involvement, such as The Leap Frog Group, may ultimately improve this process. 